\title{
Case-control study of risk factors for disease in the neck and shoulder area
}

\author{
K Ekberg, B Björkqvist, P Malm, B Bjerre-Kiely, M Karlsson, O Axelson
}

\begin{abstract}
A case-control study was performed to elucidate the strength of the relation between musculoskeletal disorders in the neck and shoulders and physical, organisational, and psychosocial aspects of the work environment. Cases were identified as those persons who consulted a physician in a community in southern Sweden for new musculoskeletal disorders in the neck and shoulders during a study period from August 1988 to the end of October 1989. One hundred and nine cases were collected and clinically examined. The cases also answered the Nordic questionnaire on symptoms as well as a questionnaire on work conditions and background factors. Controls were drawn as a random sample of the working population in the community where the cases appeared. A total of 637 controls answered the same questionnaires as the cases. Odds ratios (ORs) were calculated by logistic regression. The odds ratios were 11.4 for women, 4.9 for immigrant background, and 3.7 for current smoking. To exercise rarely, compared with often, appeared as a preventive factor with an $O R$ of $0 \cdot 3$. The ORs for various determinants of physical work load were 7.5 for repetitive movements demanding precision, 13.6 for light lifting, 3.6 for uncomfortable sitting positions, 4.8 for work with lifted arms, and 3.5 for a rushed work pace. Regarding work organisational determinants, the ORs were 16.5 for ambiguity of work role (uncertainty whether the person could manage the work) $\mathbf{2 . 6}$ for low quality work, and 3.8 for high demands on attention. Several of the determinants showed a significant dose-response relation with disease. It seems that work organisation and psychosocial work conditions are as important determinants for disease in the neck and shoulders as are the physical work conditions.
\end{abstract}

(Occup Environ Med 1994;51:262-266)

The relation between the development of musculoskeletal disorders in the neck, shoulders, and upper limbs and strained workload has become a subject of growing interest during the past decade. As well as the suffering of the individual person there are considerable economic costs to the community due to sick leaves. Some organisational and ergonomic work conditions such as repetitive tasks, force, static load, extreme joint positions, high work pace, poor psychosocial work environment and low job satisfaction seem to be determinants for development of symptoms. ${ }^{1-6}$ Brisson et $a l^{6}$ found strong associations between disability due to musculoskeletal disorders and duration of employment in piecework. A pronounced type A behaviour among blue collar workers has also been associated with a higher incidence of musculoskeletal symptoms. ${ }^{7}$ Dimberg et al found that the duration of absenteeism due to symptoms in the neck and upper extremities increased with increasing physical strain in the job. ${ }^{8}$ The symptoms seem to persist at least over a period of three years after retirement from heavy physical workload. ${ }^{9}$

Most of the studies mentioned are cross sectional in character and based on prevalence of symptoms and signs in various professional groups. Therefore they do not show the temporal relations between exposure and outcome. The similar results in several studies may nevertheless be considered tentative evidence for causal associations.

Hagberg and Wegman have reviewed studies based on clinical examinations of the cases in different occupational groups. ${ }^{10}$ They found that the associations obtained between job characteristics and disability suggest that highly repetitive shoulder muscle contractions, static contractions, and work at shoulder level are hazardous exposure factors. Work organisation and psychosocial conditions at work were not investigated in these clinical studies, and there is a need to consider multifactorial determinants of musculoskeletal disease. It should be noted also that an increased occurrence of symptoms in certain work tasks does not necessarily mean that these work conditions are generating disease in a clinical sense.

The aim of our case-control study was to elucidate the strength of the relation between disease in the neck and shoulder area and physical as well as organisational and psychosocial aspects of the work environment.

\section{Material and methods}

Our study was performed in a semirural community in southern Sweden. The labour market is characterised by many small manufacturing companies and one large rubber industry. Piecework is common. The Industrial Health Care Unit and the District 
Health Care Unit cover the primary health care needs in the area except for rubber workers, who had access to the health care facilities of the industry. The study period was from August 1988 to the end of October 1989 (15 months).

\section{SUBJECTS}

The common inclusion criteria applied were: (1) an age range between 18 and 59 years; (2) at least two months occupational experience of the last held job and occupationally active at $100 \%$ of the scheduled work times during at least four of the past six months. For the controls these criteria were formulated as a yearly income of at least SEK 45000 (about 8000 US dollars) and not having been on sick leave for more than two months during the past six months.) (3) not employed at the large rubber industry in the area. The criterion (2) was established to avoid the chronically ill and occupationally unstable people. The third criterion was established to avoid problems from special health care conditions at the single large company in the area compared with the many small industries.

\section{Cases}

The cases were taken as those who consulted a physician in the community for musculoskeletal disorders in the neck, shoulder, arm, or upper thorax during the study period. To finally qualify as a case, the person also should have become ill immediately before seeing the physician and should at most have been on sick leave for four weeks. Those who already had a long history of musculoskeletal problems were not accepted as cases as the pattern of determinants might have been obscured in the course of time. Also, the disorder should not be caused by traumatic or infectious agents or by an accident. Patients with malignancy, rheumatic diseases, abuse, or pregnancy were also excluded.

All cases were examined by the same physician and physiotherapist and diagnoses were set according to predetermined criteria as suggested by Waris et al. ${ }^{11}$ Cases were then grouped according to their main diagnosis into three groups: (1) disorders in the neck and shoulders, (2) disorders in arms and elbows, and (3) disorders in the hands and wrists. In our paper we present only cases with their main diagnosis in the neck and shoulders (group (1)), as the determinants for the different disease groups are likely to differ.

\section{Controls}

One thousand people living in the same area as the cases and who would have seen the same physicians if ill, were randomly selected for the study. We used the Swedish insurance register after approval of the local ethics committee. A stratified sample of $10 \%$ of the population was drawn, based on geographic area so that each stratum corresponded to $10 \%$ of the population that belonged to each health care unit, in all 900 subjects. The remaining 100 were saved for substitution if necessary. A letter of introduc- tion about the study was mailed to these 900 people in the middle of the study period, followed by a mailed questionnaire a week later. In all, 26 people in the sample had moved from the community and one had died. These subjects were replaced with 27 new subjects, randomly drawn from the remaining 100 people of the primary sample. Due to limited funds the controls were not examined clinically. If they had become ill during the study period, however, they would have appeared at the health care units.

\section{QUESTIONNAIRE}

All subjects answered a modified version of the Nordic questionnaire on musculoskeletal symptoms. ${ }^{12}$ In the questionnaire the subjects stated whether they had had symptoms during the past six months. They also answered a questionnaire on their work situation, physical demands at work, work organisation, and psychosocial work conditions. The background factors, age, sex, ethnic background, family situation, smoking habits, and exercise habits were also included.

\section{MEASURES OF EXPOSURE}

Measures of exposure are based on the information given in the questionnaire. Statistical analysis of the exposure questions has been described in detail elsewhere. ${ }^{5}$ Briefly, 20 items on physical work conditions were reduced to seven factors by factor analysis. The seven finally considered determinants (factors) were: (1). uncomfortable sitting position, (2) uncomfortable standing position, (3) physically demanding work, (4) light lifting $(<6 \mathrm{~kg})$, (5) repetitive movements demanding precision, (6) work with lifted arms, and (7) monotonous work position. Each body position was described by a graph in the questionnaire. The rating scales, ranging from one to four, were based on average duration in hours per day of each item of exposure. Physically demanding work (heavy lifting) was defined by weights of objects $(6-12,12-30,>30 \mathrm{~kg})$ and how often they were lifted. Fifty two items on organisational and psychosocial work conditions were reduced by factor analysis to eight factors describing (1) psychological work climate, (2) quality of work content, (3) work pace, (4) demands on attention, (5) work planning, (6) job security, (7) job constraints, and (8) work role ambiguity. Each item was rated on a scale ranging from 1 (not at all) to 7 (to a large extent).

Background determinants that could have been of importance for development of musculoskeletal disorders and hence affect the associations between disease and work environment were also included. The determinants were age, sex, ethnic background (born or not born in Sweden), smoking habits, exercise habits, and having preschool children. 
coded into tertiles based on the distributions of the cases. Because the cases were likely to be more highly exposed in at least some of the exposure factors, the distributions of the cases were used to make sure that the high exposure categories would be covered. The lowest $30 \%$ were coded as 1 , the middle $40 \%$ were coded as 2 , and the highest $30 \%$ of the exposure distributions were coded as 3 .

Odds ratios (ORs) based on multiple logistic regression and confidence intervals at the $90 \%$ level (90\% CIs) were calculated. Two control groups were established: (1) control group A consisting of those who, according to the questionnaire, were free of musculoskeletal symptoms in all parts of the body and (2) control group B consisting of control group A plus those subjects who had indicated some musculoskeletal symptoms in other parts than the neck and shoulder area.

The lowest levels of exposure were used as reference for the risk factors. For the various background determinants, age was coded into

Table 1 Demographic data of the study groups

\begin{tabular}{llll}
\hline Determinant & $\begin{array}{l}\text { Cases } \\
(\%, n=109)\end{array}$ & $\begin{array}{l}\text { Control group } A \\
(\%, n=136)\end{array}$ & $\begin{array}{l}\text { Control group } B \\
(\%, n=327)\end{array}$ \\
\hline Sex (female) & 80 & 39 & 39 \\
Immigrants & 28 & 4 & 8 \\
Current smokers & 62 & 23 & 25 \\
Preschool children & 23 & 23 & 19 \\
Type of employment: & & 28 & 29 \\
$\quad$ Blue collar & 77 & 19 & 29 \\
Service, health care & 18 & 53 & 49 \\
White collar & 5 & & \\
\hline
\end{tabular}

Table 2 Odds ratios (ORs) and 90\% CIs for determinants for diseases in the neck and shoulders $(n=109)$.

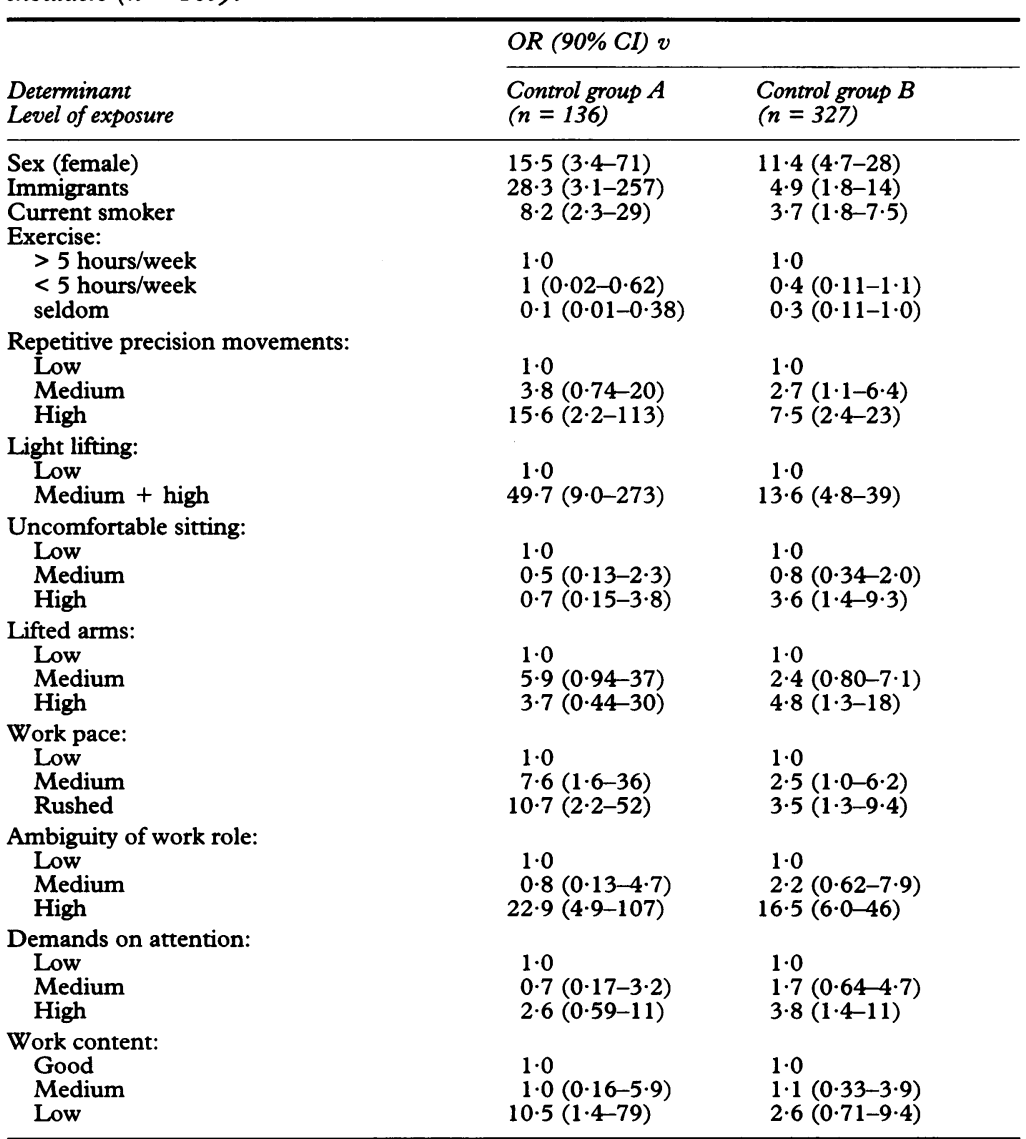

decades and the youngest age group constituted the reference for age, males were used as the reference for sex, Swedes as the reference for immigrants, non-smokers as the reference for smokers, and the highest level of leisure time exercise (at least five hours per week) was the reference for exercise. Not having preschool children was the reference for having preschool children.

\section{Results}

Each case obtained a main diagnosis and in some cases also additional diagnoses. Altogether 109 subjects had a main diagnosis of neck or shoulder disorders. The most common diagnoses were cervical syndrome or rhizopathi (18\%), tension neck syndrome (47\%), and humeral tendinitis $(27 \%)$.

The response rate was $73 \%$ for the controls (655 subjects responded). Eighteen subjects were excluded as they did not fulfil the occupational criteria (due to studies, military service, etc). The final group therefore comprised 637 persons. As the control group was obtained as a random sample of the population it is not surprising that many reported symptoms from the neck (43\%) and shoulders $(32 \%)$, and also from other parts of the body. One hundred and thirty six controls $(21 \%)$ did not report any musculoskeletal symptoms in any part of the body.

Table 1 shows the demographics of the groups. The median age of the cases was 39 (range 18-57) years. The age distribution of the control groups were similar (control group A: 38 (18-58) years, control group B: 39 (18-59) years). The average working hours were 36 hours among the cases and 38 hours in both control groups.

Table 2 shows ORs and $90 \%$ CIs for disease in the neck and shoulders. To make the results easier to comprehend, only determinants with significant associations in relation to one or both of the control groups are presented.

Female sex and immigrant background were associated with diseases in the neck and shoulder area. To be a smoker was also a significant determinant. For exercise, less than five hours per week seemed preventive whereas more extensive exercise, at least five hours per week, was significantly associated with disease in the neck and shoulders. Age and having preschool children were not associated with disease.

Repetitive movement demanding precision was a significant physical determinant with a dose-response relation showing higher risks for higher degrees of exposure. Light lifting was also a strong determinant for disease. The medium and high exposure categories were combined for this factor as there were too few cases in one of the exposure categories. Long durations of uncomfortable sitting and work with lifted arms were significant determinants compared with the larger control group B. To work standing in uncomfortable positions, monotonous work positions, and physically demanding work 
(heavy lifting) were not significant determinants for disease in the neck and shoulders.

Quantitative demands expressed as self rated work pace had a significant doseresponse relation with diseases in the neck and shoulders. Work role ambiguity - that is, uncertainty about how to best perform the work and uncertainty about whether the person could manage the work-was a strong determinant in the high exposure category. High demands on attention also seemed to be a determinant when compared with control group B. Low quality work-namely, lack of stimulation and variation in the job, low opportunities for development, and a low job discretion-were significant determinants when compared with the controls with no symptoms (control group A). Deficits in the psychological work climate, work planning, job security, and job constraints were not significant determinants for disease in the neck and shoulders.

As shown in table 2, there are distinct dose-response relations between disease in the neck and shoulders and some determinants-namely, repetitive precision movements, work pace, and low quality work-irrespective of control group. When compared with the larger control group B, work with lifted arms, work role ambiguity, and demands on attention also showed distinct dose-response relations with disease.

\section{Discussion}

There are few studies of musculoskeletal diseases in the neck and shoulders where the outcome variable is based on clinical examinations. In this study attempts were made to follow distinct diagnostic criteria as presented by Waris et al. ${ }^{11} \mathrm{~A}$ diagnosis in the neck and shoulder area was, however, often accompanied by other, related disorders. To overcome this problem the different diagnoses were combined into a more general neck and shoulder disease entity in the analyses.

A problem in epidemiological studies on musculoskeletal disorders is the assessment of exposure to work loads. In some studies exposure has been approximated by job title. ${ }^{10} \mathrm{~A}$ job title, however, may comprise many different tasks and indicate a huge variation in exposure to work loads. To properly identify determinants more specific measurements of exposure are needed. In many studies information about exposure to various factors is collected by questionnaires. An implicit assumption is then that subjects have been in this exposure for some time. Given well defined exposure categories and simple, concrete questions, a questionnaire may give rough but reasonable information on exposure conditions. In our study, dose-response relations were obtained for several determinants, which strengthens the credibility of the exposure estimates. Inclusion criteria were set to ensure that the same exposure should have been present for at least some months. This may be considered a short time, but disease in the neck and shoulders may develop fast in some subjects if work conditions are adverse. ${ }^{13}$ To capture the early stages of disease, comparatively short durations of exposure had to be accepted.

The advancement of automation and specialisation in work, usually with a generally reduced physical workload and a reduction of heavy lifting, have created work tasks with fast work rates and a high degree of repetitiveness. Small industries of the type that generated many of the cases in this study, often have high demands on productivity to survive. They have generally become effective by adapting the work organisation to the equipment. The tasks have become impoverished both physically and mentally, but the quantitative demands are high and based on piece wages. The results clearly underline these organisational problems. Repetitive movements demanding precision often also comprise lifting of small pieces from one position to another in the manufacturing industries. Exposure to this kind of work load is the most powerful physical risk in our study and comparable with results from other studies. ${ }^{1-5} 10$ Work pace shows a pronounced dose-response relation with musculoskeletal disorders in this study. Piece wages, common for workers in the manufacturing industries, have been reported by others ${ }^{6}$ to contribute to high work pace and to promote subjective strain.

The increased risk of disease due to low quality work content - that is, lack of stimulation and variation in the work tasks, low opportunities for development, and low job discretion-may be considered to reflect other aspects of the same work organisation principle that was creating physical risks. These characteristics of a work situation have in many studies turned out to be significant risk factors for stress related cardiovascular diseases, hence indicating that psychological mechanisms also may be mediating musculoskeletal disorders to some degree. Psychological demands and lack of possibility to talk to work mates were associated with muscle tension and emotional states in a study by Theorell et al, ${ }^{14}$ supporting the assumption of a relation between psychosocially adverse job conditions and symptoms from the locomotor system.

Work role ambiguity-that is, uncertainty about how to best perform the work and uncertainty about whether the person can manage the tasks-is a powerful determinant for disease in the neck and shoulder area. Work role ambiguity may have its origin in lack of feedback on performance and vague or muddled instructions from the foremen or other superiors. Also, inappropriate training for the job, or training that is not adapted to the characteristics and background of the employees, may cause uncertainties about how to manage the work.

Being a woman and being an immigrant was highly associated with disease in the neck and shoulder area. Although these two characteristics are commonly associated with risk, only a few studies include sex and ethnic 
background in the analyses for possible determinants. Dimberg et $a l^{8}$ found that women in jobs with high strain on the neck and upper extremities were sicklisted twice as often as men in the same type of job. Kvarnström ${ }^{15}$ found that occupational shoulder disorders occurred 10 times more often in women than in men in a large manufacturing industry. There was an over-representation of women in the jobs with the highest risks, which may be part of the explanation. Another suggested explanation was lower muscle strength in women. Others, however, have found little support for physical strength protecting against musculoskeletal disorders. ${ }^{4}$ Exercise, which could be assumed to improve physical strength, seemed to be a risk factor in our study.

Kvarnström ${ }^{15}$ also compared Swedes and immigrants in jobs with high risk for occupational shoulder disorders and found a relative risk for immigrants of $\mathbf{4 . 9}$ for assembly line operators and 3.3 for winders. He suggested that immigrants may have used faulty work techniques owing to lack of education and instruction, or had difficulties in changing job when symptoms began to occur, due to the language barrier. It is conceivable that similar reasons exist in our study, and also that being an immigrant is associated with the experience of work role ambiguity.

Smokers were strongly over-represented among the cases in this study. To be a smoker was significantly associated with disease in the neck and shoulders. In a cross sectional study, Tsai et $a l^{16}$ also found smoking to be one factor for increased risk of musculoskeletal injury (relative risk $=1 \cdot 23$ ). The effect of smoking on muscles, tendons, and nerves is not clear. It has been suggested for low back pain that the nicotine induced vasoconstriction of small nutrient vessels may reduce the blood flow to the intervertebral discs, thereby rendering them more vulnerable to injury. ${ }^{16}$ Similar mechanisms could operate for the neck and shoulders.

Control group A represents a group of people with no musculoskeletal symptoms. As expected, a comparison with this group in general gives higher risk estimates than comparisons with the other control group which comprised subjects with musculoskeletal symptoms in other parts of the body. Although the $90 \%$ CIs for most of the factors overlap, it seems that some determinants are particularly strong when compared with group A. These factors would be those that are of greatest interest to focus on in preventive work. Light lifting, high work pace, work role ambiguity, and a low quality of work seem to be those determinants that should be given primary attention.
To conclude, musculoskeletal disease in the neck and shoulders seems to have a multifactorial origin. The significant determinants constitute an aggregate of complex organisational and physical factors. Repetitive precision movements and light lifting often make a work situation that also has certain organisational features, such as a high work pace, impoverished work content, minimal training, and lack of feedback on performance. Improvements of just one of the determinants may not necessarily lead to improved health of the workers, as the remaining determinants may preserve the strenuous situation. Rather, our results indicate that preventive efforts should focus on the entire complex that constitutes the work situation.

This study was supported by the Swedish Work Environment Fund, which is gratefully acknowledged.

1 Tola S, Riihimäki $H$, Videman $T$, Viikari-Juntura $E$, Hänninen $K$. Neck and shoulder symptoms among men in machine operating, dynamic physical work and in machine operating, dynamic physical work and 14:299-305.

2 Ohlsson K, Attewall R, Skerfving S. Self-reported symptoms in the neck and upper limbs of female assembly workers. Scand ₹ Work Environ Health 1989;15:75-80.

3 Linton S. Risk factors for neck and back pain in a working population in Sweden. Work and Stress 1990;4:41-9.

4 Westgaard RH, Jansen T. Individual and work related factors associated with symptoms of musculoskeletal complaints. II Different risk factors among sewing machine operators. Br f Ind Med 1992;49:154-162.

5 Ekberg K, Björkqvist B, Malm P, Bjerre-Kiely B, Karlsson $\mathrm{M}$, Axelson O. Cross-sectional study of risk factors for symptoms in the neck-shoulder area. Ergonomics 1994 (in press)

6 Brisson C, Vinet A, Vézina M, Gingras S. Effect of duration of employment in piecework on severe disability among female garment workers. Scand $\mathcal{f}$ Work Environ Health 1989;15:329-34.

7 Flodmark BT, Aase G. Musculoskeletal symptoms and type A behaviour in blue collar workers. $\mathrm{Br} \mathcal{F}$ Ind Med 1992;49:683-7.

8 Dimberg L, Olafsson A, Stefansson E, et al. Sickness absenteeism in an engineering industry-an analysis with special reference to absence for neck and upper extremity symptoms. Scand $\Im$ Soc Med 1989;17:77-84.

9 Berg M, Sandén A, Torell G, Järvholm B. Persistence of musculoskeletal symptoms: a longitudinal study. Ergonomics 1988;31:1281-5.

10 Hagberg M, Wegman DH. Prevalence rates and odds ratios of shoulder-neck diseases in different occupational groups. Br $\mathcal{F}$ Ind Med 1987;44:602-10.

11 Waris P, Kuorinka I, Kurppa K, et al. Epidemiologic screening of occupational neck and upper limb disorders: methods and criteria. Scand $f$ Work Environ Health 1979;5:25-38.

12 Kourinka I, Jonsson B, Kilbom $\AA$, et al. Standardised Nordic questionnaires for the analysis of musculoskeletal symptoms. Appl Ergonomics 1987;3:233-7.

13 Maeda $\mathrm{K}$, Horiguchi S, Hosokawa M. History of the studies on occupational cervicobrachial disorder in Japan and remaining problems. $₹$ Human Ergol 1982;11: and reme.

14 Theorell $T$, Harms-Ringdahl $K$, Ahlberg-Hultén G, Westin B. Psychosocial job factors and symptoms from the locomotor system - a multicausal analysis. Scand $\mathcal{F}$ Rehabil Med 1991;23:165-73.

15 Kvarnström S. Occurrence of musculoskeletal disorders in a manufacturing industry, with special attention to occupational shoulder disorders. Scand 7 Rehabil Med 1983;(suppl 8):6-35.

16 Tsai S, Gilstrap EL, Cowles SR, Waddell LC, Ross CE. Personal and job characteristics of musculoskeletal injuries in an industrial population. $f$ Occup Med 1992;34:606-12. 